\title{
On Generalized Gaussian Quadrature *
}

\author{
By Yudell L. Luke, Bing Yuan Ting and Marilyn J. Kemp
}

\begin{abstract}
A general interpolation formula is derived such that when it is multiplied by a weight function and integrated, the result becomes a generalized Gaussian quadrature scheme which allows for an arbitrary number of preassigned nodes. The errors in the interpolation and quadrature formulas are studied. All of this generalizes previous results of the first-named author where no preassigned nodes were permitted.
\end{abstract}

I. Introduction. In a previous paper, Luke [1] showed that if a set of polynomials satisfies an orthogonality relation with respect to integration, the set also satisfies an orthogonality relation with respect to summation. The orthogonality relations give rise to two representations of an arbitrary function $f(x)$ in series of the orthogonal polynomials $q_{n}(x)$. One is infinite with coefficients $c_{k}$; the other is composed of a finite number of terms, say $n+1$, with coefficients $d_{k, n}$. A connection between these coefficients is established. The error in the finite interpolation formula is characterized in terms of the coefficients $c_{k}$. Integration of the finite interpolation formula leads to the Gaussian quadrature formula and the error in this formula is expressed in the form $\Sigma_{s=0}^{\infty} c_{2 n+2+s} g_{2 n+2+s}$ where the coefficients $g_{k}$ depend only on the system of orthogonal polynomials and the associated weight function. Closed form expressions for the early $g_{k}$ 's are given in the cases where the orthogonal polynomials are those of Jacobi, Laguerre, Hermite and Bessel.

The purpose of this paper is to extend the results noted above by developing a generalized interpolation formula from which we derive a generalized Gaussian quadrature scheme to include an arbitrary number of preassigned nodes. If $(a, b)$ is the interval of integration and if the preassigned node(s) is at $a$ or at both $a$ and $b$, then the integration formulas often go by the names of Radau and Lobatto, respectively. The error analyses noted above are applied to the generalized case. A shortcoming of the previous paper is the lack of numerical examples to illustrate use of the error formulae. In this paper, we develop several numerical examples to manifest the efficiency of the error formulas for Gaussian, Radau and Lobatto quadrature schemes.

This paper assumes familiarity with [1]. We restate the main results of that paper without giving proofs.

II. Some Basic Equations. Let

$$
q_{n}(x)=\sum_{k=0}^{n} a_{k, n} x^{k}
$$

Received September 17, 1973; revised October 22, 1974.

AMS (MOS) subject classifications (1970). Primary 65D30, 65D05; Secondary 33A65, $41 \mathrm{A05}, 41 \mathrm{~A} 55$.

${ }^{*}$ This research work was sponsored by the Air Force Office of Scientific Research under Grants 71-2127 and 73-0469. 
be a set of orthogonal polynomials such that

$$
\begin{gathered}
\int_{a}^{b} W(x) q_{n}(x) q_{r}(x) d x=h_{n} \delta_{n r}, \\
\delta_{n r}=0 \quad \text { if } n \neq r, \quad \delta_{n r}=1 \quad \text { if } n=r . \\
W(x)=w(x) \Omega_{m}(x), \quad \Omega_{m}(x)=\prod_{i=1}^{m}\left(x-a_{i}\right),
\end{gathered}
$$

where $W(x)$ and $w(x)$ are one signed for $a \leqslant x \leqslant b$. These polynomials satisfy the three term recurrence formula

$$
\begin{gathered}
q_{n+1}(x)=\left(A_{n} x+B_{n}\right) q_{n}(x)-C_{n} q_{n-1}(x), \quad n \geqslant 0, \\
A_{n}=\frac{a_{n+1, n+1}}{a_{n, n}}, \quad C_{n}=\frac{A_{n} h_{n}}{A_{n-1} h_{n-1}}, \quad C_{0}=0, \\
B_{n}=A_{n}\left(r_{n+r}-r_{n}\right), \quad r_{n}=\frac{a_{n-1, n}}{a_{n, n}}, \quad n>0, r_{0}=0 .
\end{gathered}
$$

We also have the Christoffel-Darboux formula

$$
\sum_{k=0}^{n} h_{k}^{-1} q_{k}(x) q_{k}(y)=\left(A_{n} h_{n}\right)^{-1} \frac{q_{n+1}(x) q_{n}(y)-q_{n}(x) q_{n+1}(y)}{x-y}
$$

We point out that the results given here reduce to those given in [1] if we now put $m=0$. However, we must caution the reader that the notations $q_{n}(x), A_{n}, B_{n}, C_{n}$, etc. are used in both places. Thus the reader will understand the quantities have different values in the two contexts unless indeed $m=0$.

III. The Interpolation Formulas. Let $f_{n+m}(x)$ be the polynomial of degree $n+m$ such that

$$
f\left(x_{k}\right)=f_{n+m}\left(x_{k}\right), \quad k=0,1, \ldots, n, \quad q_{n+1}\left(x_{k}\right)=0,
$$

$$
f\left(a_{j}\right)=f_{n+m}\left(a_{j}\right), \quad j=1,2, \ldots, m .
$$

Then by use of the Lagrangian interpolation formula and the Christoffel-Darboux formula,

$$
d_{k, n}=\frac{A_{n} h_{n}}{h_{k}} \sum_{i=0}^{n} \frac{q_{k}\left(x_{i}\right) f\left(x_{i}\right)}{\Omega_{m}\left(x_{i}\right) q_{n}\left(x_{i}\right) q_{n+1}^{\prime}\left(x_{i}\right)} .
$$

Let

$$
V_{j, k}^{(n)}=\frac{A_{n} h_{n}}{h_{k}} \sum_{i=0}^{n} \frac{q_{j}\left(x_{i}\right) q_{k}\left(x_{i}\right)}{q_{n+1}^{\prime}\left(x_{i}\right) q_{n}\left(x_{i}\right)}
$$


Then Luke [1] proved that

$$
V_{j, k}^{(n)}=\delta_{j k}, \quad j \leqslant n, \quad k \leqslant n .
$$

We also have the following properties:

$$
\begin{array}{ll}
h_{k} V_{j, k}^{(n)}=h_{j} V_{k, j}^{(n)} & \text { for all } j \text { and } k, \\
V_{j, n+1}^{(n)}=V_{n+1, k}^{(n)}=0 & \text { for all } j \text { and } k, \\
V_{n+2, k}^{(n)}=-C_{n+1} V_{n, k}^{(n)} . &
\end{array}
$$

If $a=-b$ and $q_{n}(x)$ is even or odd with $n$ even or odd, respectively, then

$$
\begin{aligned}
& V_{j, k}^{(n)}=0 \text { if } j+k \text { is odd, for all } n, \\
& V_{j, k}^{(n)}=\frac{2 A_{n} h_{n}}{h_{k}} \sum_{\gamma=0}^{[n / 2]} \frac{q_{j}\left(x_{\gamma}\right) q_{k}\left(x_{\gamma}\right)}{q_{n+1}^{\prime}\left(x_{\gamma}\right) q_{n}\left(x_{\gamma}\right)}
\end{aligned}
$$

if $j+k$ is even and $n=2 r-1$ is odd, and

$$
V_{j, k}^{(n)}=\frac{2 A_{n} h_{n}}{h_{k}} \sum_{\gamma=0}^{n / 2} \frac{q_{j}\left(x_{\gamma}\right) q_{k}\left(x_{\gamma}\right)}{q_{n+1}^{\prime}\left(x_{\gamma}\right) q_{n}\left(x_{\gamma}\right)}+\frac{A_{n} h_{n} q_{j}(0) q_{k}(0)}{h_{k} q_{n+1}^{\prime}(0) q_{n}(0)}
$$

if $j+k$ and $n$ are both even.

A useful result is a recursion formula for $V_{n+r, k}^{(n)}$.

$$
\begin{aligned}
& V_{n+r, k}^{(n)}=\left(B_{n+r-1}-\frac{A_{n+r-1} B_{k}}{A_{k}}\right) V_{n+r-1, k}^{(n)}-C_{n+r-1} V_{n+r-2, k}^{(n)} \\
&+ \frac{A_{n+r-1} h_{k+1}}{A_{k} h_{k}} V_{n+r-1, k+1}^{(n)}+\frac{A_{n+r-1} h_{k-1} C_{k}}{A_{k} h_{k}} V_{n+r-1, k-1}^{(n)}, k \geqslant 0, \\
& V_{n+r-1,-1}^{(n)}=0,
\end{aligned}
$$

$$
V_{n+r, n-r+2}^{(n)}=-\frac{A_{n+1} A_{n+2} \cdots A_{n+r-1} h_{n+1}}{A_{n-r+2} A_{n-r+3} \cdots A_{n} h_{n-r+2}}, \quad r \geqslant 2 .
$$

In our later work, we have need for the coefficients $V_{2 n+2+s-k, k}^{(n)}$ for $k=0,1, \ldots$, $n$ and $s=0,1,2, \ldots$ which follow from (19)-(21).

We consider

$$
\Omega_{m}(x) F(x)=f(x)-q_{n+1}(x) \sum_{j=1}^{m} \frac{\Omega_{m}(x) f\left(a_{j}\right)}{\left(x-a_{j}\right) \Omega_{m}^{\prime}\left(a_{j}\right)}
$$

and suppose that $F(x)$ can be represented by an expansion in series of the orthogonal polynomials $q_{k}(x)$ which is uniformly convergent in $[a, b]$. Thus

$$
\begin{gathered}
F(x)=\sum_{k=0}^{\infty} c_{k} q_{k}(x), \\
c_{k}=h_{k}^{-1} \int_{a}^{b} \Omega_{m}(x) w(x) F(x) q_{k}(x) d x .
\end{gathered}
$$


Clearly

$$
f\left(x_{k}\right)=\Omega_{m}\left(x_{k}\right) \sum_{j=0}^{\infty} c_{j} q_{j}\left(x_{k}\right) .
$$

Put this in (10) and so obtain the important relation

$$
d_{k, n}=c_{k}+\sum_{s=0}^{\infty} c_{2 n+2+s-k} V_{2 n+2+s-k, k}^{(n)} .
$$

Next we give an interesting expansion for the error in the interpolation formula (8). From (8)-(10), (22), (23) and (26), we have

$$
R_{n+m+1}(x)=\Omega_{m}(x) \sum_{k=1}^{\infty} c_{n+k}\left\{q_{n+k}(x)-\sum_{j=0}^{k-2} V_{n+k, n-j}^{(n)} q_{n-j}(x)\right\} ;
$$

and in view of the properties of $V_{j, k}^{(n)}$, we get

$$
\begin{aligned}
R_{n+m+1}(x)=\Omega_{m}(x)\left\{c_{n+1} q_{n+1}(x)+c_{n+2}\left[q_{n+2}(x)+c_{n+1} q_{n+1}(x)\right]\right. \\
+c_{n+3}\left[q_{n+3}(x)+\frac{A_{n+2} A_{n+1} h_{n+1}}{A_{n} A_{n-1} h_{n-1}} q_{n-1}(x)\right. \\
\left.\left.\quad-C_{n+1}\left(\frac{A_{n+2} B_{n}}{A_{n}}-B_{n+2}\right) q_{n}(x)\right]+\ldots\right\},
\end{aligned}
$$

and finally with the aid of (4),

$$
R_{n+m+1}(x)=\Omega_{m}(x) c_{n+1} q_{n+1}(x)\left[1+\frac{c_{n+2}}{c_{n+1}}\left(A_{n+1} x+B_{n+1}\right)+\ldots\right] \text {. }
$$

IV. Generalized Gaussian Quadrature Formula. Multiply (8) by $w(x)$ and integrate from $a$ to $b$, and so obtain

$$
\begin{aligned}
\int_{a}^{b} w(x) f(x) d x & =\sum_{k=0}^{n} \lambda_{k, n}^{(m)} f\left(x_{k}\right)+\sum_{j=1}^{m} \mu_{j, m}^{(n)} f\left(a_{j}\right)+S_{2 n+m+2}, \\
\lambda_{k, n}^{(m)} & =\frac{A_{n} h_{n}}{\Omega_{m}\left(x_{k}\right) q_{n}\left(x_{k}\right) q_{n+1}^{\prime}\left(x_{k}\right)}, \\
\mu_{j, m}^{(n)} & =\left(\Omega_{m}^{\prime}\left(a_{j}\right) q_{n+1}\left(a_{j}\right)\right)^{-1} \int_{a}^{b} \frac{w(x) \Omega_{m}(x) q_{n+1}(x)}{x-a_{j}} d x, \\
S_{2 n+m+2} & =\int_{a}^{b} w(x) R_{n+m+1}(x) d x .
\end{aligned}
$$

This is the generalized Gaussian quadrature formula. When $m=0$ it reduces to the Gaussian quadrature formula. By using arguments analogous to those of Krylov [2] , it could be shown that (30) has degree of precision $2 n+m+1$.

We now show how to express the error in terms of the coefficients $c_{n}$. Using equations (8)-(10), (22), (23), and (30), we obtain 


$$
\begin{aligned}
S_{2 n+m+2} & =\int_{a}^{b} w(x)\left(f(x)-f_{n+m}(x)\right) d x \\
& =\sum_{k=0}^{\infty} c_{k} \int_{a}^{b} w(x) \Omega_{m}(x) q_{k}(x) d x-\sum_{k=0}^{n} d_{k, n} \int_{a}^{b} w(x) \Omega_{m}(x) q_{k}(x) d x \\
& =\frac{h_{0}}{a_{0,0}}\left(c_{0}-d_{0, n}\right),
\end{aligned}
$$

by virtue of orthogonality, and in view of (26), it follows that

$$
\begin{gathered}
S_{2 n+m+2}=\sum_{s=0}^{\infty} c_{2 n+2+s} g_{2 n+2+s} \\
g_{2 n+2+s}=-\frac{h_{0}}{a_{0,0}} V_{2 n+2+s, 0}^{(n)} .
\end{gathered}
$$

The error formula is quite interesting in that the $c_{n}$ 's depend on $f(w), w(x)$ and the system of orthogonal polynomials while the $g_{n}$ 's depend only on the system of orthogonal polynomials. Following the procedure developed by Luke [1], we can write the $g_{n}$ 's in terms of the coefficients $A_{n}, B_{n}, C_{n}$ and $h_{n}$. Thus from (21),

$$
g_{2 n+2}=\frac{A_{n+1} A_{n+2} \cdots A_{2 n+1} h_{n+1}}{A_{0} A_{1} \ldots A_{n} a_{0,0}}
$$

and further coefficients can be deduced with the aid of the recursion formula (19). For details, see the reference cited.

V. Additional Comments. In this section we consider some aspects of our representation for the error, and then compare our approach with that of other workers.

In our work we tacitly assume $w(x)$ and $f(x)$ are given in analytic form. Further, the form of $f(x)$ is of such complexity that an exact representation for the coefficients $c_{n}$ from which numerical values are readily extracted is not available. This, of course, is to be expected, for otherwise we would have no problem as $c_{0}$ is the desired integral. If $w(x)$ is known and $f(x)$ is given by experimental data, there is no need to resort to sophisticated integration formulas and approximations based on piecewise continuous line segment approximations to $f(x)$ are about the best one can do. It should be clear that use of our integration formulas and attendant error analyses require some mathematical sophistication. The processes are designed for high level accuracy and realistic estimates of the error (see numerical examples).

We now discuss related analyses of other workers. If $f(x)$ is analytic on and within a completely closed simple contour $C$ which has the segment $[a, b]$ in its interior, then it is known that the error in the Gaussian quadrature formula can be expressed as a contour integral. Thus with $m=0$,

$$
\begin{gathered}
S_{2 n+2}=(2 \pi i)^{-1} \int_{C} \frac{j_{n}(z) f(z)}{q_{n}(z)} d z, \\
j_{n}(z)=\int_{a}^{b} \frac{w(t) q_{n}(t)}{z-t} d t,
\end{gathered}
$$


and both $j_{n}(z)$ and $q_{n}(z)$ satisfy the same recurrence formula. This representation was first introduced by Barrett [3]. For the case of the Jacobi polynomials ( $a=-1$, $b=1)$, he uses an asymptotic representation for $j_{n}(z) / q_{n}(z)$ and under the assumption that $f(z)$ is analytic on and within a certain ellipse with foci at $z= \pm 1$ except for poles on this ellipse, he obtains an asymptotic estimate for $S_{2 n+2}$. The Gauss-Laguerre and Gauss-Hermite quadratures are also discussed. Analyses similar to that of Barrett for Gauss-Legendre, -Laguerre and -Hermite quadrature have been given by McNamee [4], and studies similar to that of Barrett for Gauss-Legendre and Chebyshev (first kind) quadrature have been treated by Chawla and Jain [5], [6] .

If $f(x)$ is analytic as above and is expanded in series of Legendre polynomials, Chawla [7] derives the error in the form of (32). Under the same assumptions, Basu [8] gets (26) and (32) for ordinary Gaussian quadrature $(m=0)$. However, he does not give the recursion formula for the coefficients (33).

Donaldson and Elliott [9] generalize the work of Barrett [3] and others by allowing for Radau and Lobatto quadrature and by studying the error by means of a contour integral. In general, they require that $f(z)$ is analytic on $(a, b)$ except possibly for singularities at $a$ or $b$, and $f(z)$ may have poles or branch points or both near the segment of integration. It is not our intent to summarize in detail the work of Donaldson and Elliott. Suffice it to say that both the latter workers and we are concerned with 'exact' representations of the error and have the philosophy of using asymptotic methods to estimate such representations.

As remarked, Donaldson and Elliott [9] assume $f(z)$ is analytic except for branch points and poles and represent the error by a single integral. In our treatment, we do not suppose analyticity, but do require that $F(x)$, see (22), can be represented as in (23). Our characterization of the error is in the form of an infinite series, each term of which is the product of two functions as explained in the discussion following (33). Once the system of orthogonal polynomials is specified, the $g_{k}$ 's can be evaluated independently of the function being integrated. The $c_{k}$ 's depend both on the system of orthogonal polynomials and $F(x)$. Quite often various integral representations for $c_{k}$ are available (see, for example, the work of Tuan and Elliott [10] and Luke [11]) and under appropriate conditions one form might be better for achieving asymptotic estimates than the others. This flexibility can be advantageous. It is anticipated that an asymptotic estimate of the first term of the infinite series will give sufficient information on the error.

The basic assumption in our approach, namely that $F(x)$ can be represented as described by (23) versus the assumption that $f(x)$ and so also $F(x)$ is analytic deserves further comment. If $f(x)$ is to be expanded say in series of Chebyshev polynomials of the first kind, then the assumption of analyticity is much too strong. It is sufficient that $f^{\prime}(x)$ be continuous in $[-1,1]$. On the other hand, if $f(x)$ is to be expanded in series of the Laguerre polynomials, then analyticity alone is not sufficient to guarantee the form described by (23). Criteria for the latter type representation, if known, are intimately connected with the choice of orthogonal polynomials. On the pragmatic side, best results can be expected if $f(x)$ is analytic in as large a region in the complex plane as possible. Thus, all singularities on or near the axis of integration 
should be incorporated into the weight function $w(x)$.

VI. Numerical Examples. In this section we illustrate the ideas of our paper for the examples $e^{\sigma x}, \sigma=2$ and $(1+x)^{-1}$. In view of space limitations we present some data for Gaussian quadrature only of the above functions. Further data relating to Radau and Lobatto quadrature will be found in a report with the same title as this paper which is available to the reader upon request. This report also gives a detailed theoretical analysis of formulas relating to Gaussian, Radau and Lobatto quadrature of $e^{\sigma x}$ and $(1+x)^{-1}$. In our present version, some analytical formulas are presented. When without encumbering additional space, we can give formulas more general than actually needed for the computations, we do so. It is convenient to use hypergeometric notation and other symbols commonly employed in special functions. For these and other data, see Luke [11].

Example I. $f(x)=e^{\sigma x}, \sigma=2$. Here, we consider Gaussian quadrature and so let

$$
a=-1, b=1, w(x)=1 \quad \text { whence } \alpha=\beta=0, m=0 .
$$

Thus $q_{n}(x)$ is the Legendre polynomial of order $n$. If $\alpha=\beta$, it can be deduced from Luke $[11$, v. 2 , p. 32 , Eq. (7)], that

$$
\begin{gathered}
e^{\sigma x}=\sum_{k=0}^{\infty} c_{k} P_{k}^{(\alpha, \alpha)}(x), \\
c_{k}=\frac{2(\pi / 2 \sigma)^{1 / 2}(k+\alpha+1 / 2) \Gamma(k+2 \alpha+1)}{(2 \sigma)^{\alpha} \Gamma(k+\alpha+1)} I_{k+\alpha+1 / 2}(\sigma),
\end{gathered}
$$

where $P_{n}^{(\alpha, \beta)}(x)$ is the Jacobi polynomial and $I_{\nu}(\sigma)$ is the familiar notation for the modified Bessel function. Generally, the coefficients $c_{k}$ are not in such simple form and we must resort to asymptotic estimates. For the example at hand, we follow the discussion given by Elliott [12] and Luke [11, v. 1, pp. 295, 296]. With $\alpha=\beta=0$, we get

$$
c_{k} \sim \frac{\exp (\sigma \cosh \theta-(k+1 / 2) \theta) \sinh \theta}{(\cosh \theta)^{1 / 2}}, \quad \cosh \theta=\left(1+k^{2} / \sigma^{2}\right)^{1 / 2} .
$$

The $c_{k}$ 's according to (38) and (39) are given in columns A and $B$, respectively, of Table 1.1.

To illustrate interpolation and quadrature, let $n=2$. The $x_{k}$ 's and $d_{k, 2}$ 's are given in Table 1.2. Values of $V_{j, k}^{(2)}$ and partial sums of the series for $d_{k, 2}-c_{k}$ for $k=0,1,2$ in accordance with (26) are given in Table 1.3. Clearly, the first term of the series is dominant.

To illustrate the interpolation formula and its error, we use (8) and (27). Let $x=0.4$. Then $f(x)=e^{0.8}=2.22554093$. We find $f_{2}(x)=2.55001$ and six terms of (27) gives the remainder -0.32447 which is correct. The first term of the series (27) gives the approximate remainder -0.29181 while the corresponding asymptotic estimate using column $B$ of Table 1.1 gives -0.27629 . The asymptotic estimate is quite reasonable considering the fact that $n$ is small and $\sigma$ is not small with respect to $n$. 
TABLE 1.1

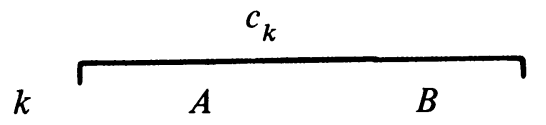

$\begin{array}{lll}0 & 1.81343020 & 0.00000000\end{array}$

$\begin{array}{lll}1 & 2.92314823 \quad 2.14967608\end{array}$

$2 \quad 1.75928044 \quad 1.57099058$

$3 \quad 0.66319766 \quad 0.62793500$

$4 \quad 0.18231535 \quad 0.17667631$

$\begin{array}{lll}5 & 0.03943333 & 0.03863618\end{array}$

$\begin{array}{lll}6 & 0.00702774 & 0.00692664\end{array}$

$\begin{array}{lll}7 & 0.00106469 & 0.00105309\end{array}$

$\begin{array}{lll}8 & 0.00014024 & 0.00013903\end{array}$

$9 \quad 0.00001634 \quad 0.00001622$

$10 \quad 0.00000171 \quad 0.00000170$

$110.00000016 \quad 0.00000016$

$12 \quad 0.00000001 \quad 0.00000001$

TABLE 1.2

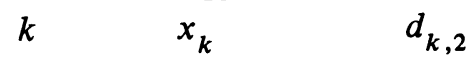

$0 \quad-0.77459666 \quad 1.81113622$

$1 \quad 0.00000000 \quad 2.90167262$

$2 \quad 0.77459666 \quad 1.62227245$

TABLE 1.3

$$
V_{6+i-k, k}^{(2)}
$$

$i k$

0

$$
1
$$
$\begin{array}{llll}0 & -0.33000000 & -0.54000000 & -0.75000000\end{array}$

$s k$

0

1

$\begin{array}{llllll}0 & -0.00231915 & -0.02129400 & -0.13673651 \\ 1 & -0.00231915 & -0.02129400 & -0.13673651 \\ 2 & -0.00229408 & -0.02148138 & -0.13698 & 248 \\ 3 & -0.00229408 & -0.02148138 & -0.13698248 \\ 4 & -0.00229397 & -0.02147565 & -0.13700904\end{array}$


For the quadrature formula (30), the weights $\lambda_{\gamma, 2}^{(0)}$ are $5 / 9,8 / 9$ and $5 / 9$ for $\gamma=0,1,2$ respectively. The approximate quadrature is 3.62227 while the exact value is 3.62686041 . Now $h_{0} / a_{0,0}=2$. Using three terms of (32) with the column $A$ data of Table 1.1, we get the error 0.00459 which is correct. The first term of this series is 0.00464 and so is dominant. If we use the first term of (32) but with the appropriate asymptotic value from column $B$ of Table 1.1, we get 0.00457 which is an excellent estimate of the error.

As a remark aside, (26) might very well be of importance in other applications. Now $d_{k, n}$ is composed of a finite sum and is readily evaluated. The manner of computing $V_{j, k}^{(n)}$ has already been indicated. Let us suppose that an asymptotic formula for $c_{r}$ is sufficiently accurate at least for $r \geqslant 2 n+2-k, k=0,1, \ldots, n$. Then (26) can be used to give improved estimates for the early $c_{k}$ 's. For a numerical illustration, see the discussion following Table 2.3.

Example II. $f(x)=(1+x)^{-1}$. Again, we consider Gaussian quadrature. This time

$$
a=0, b=1, w(x)=1 \quad \text { whence } \alpha=\beta=0, m=0,
$$

and $q_{n}(x)$ is the shifted Legendre polynomial. The shifted Jacobi polynomial is denoted by $R_{n}^{(\alpha, \beta)}(x)=P_{n}^{(\alpha, \beta)}(2 x-1)$. It is known (see Luke [11, v. 2, p. 31, Eq. (1)] ) that

$$
\begin{aligned}
& (1+x)^{-1}=\sum_{k=0}^{\infty} c_{k} R_{k}^{(\alpha, \beta)}(x),
\end{aligned}
$$

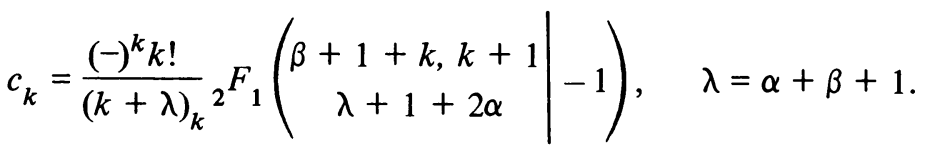

Further, the $c_{k}$ 's satisfy a three-term recurrence relation. (See Luke $[11$, v. 2, p. 159, (26) - (28)], where the parameters are so chosen to reduce the ${ }_{3} F_{2}$ to the appropriate ${ }_{2} F_{1}$ after the manner of discussion given in connection with Eqs. (63)-(66) on p. 147 of that reference.) With $x=0$ in (41),

$$
\sum_{k=0}^{\infty} \frac{(-)^{k}(\beta+1)_{k} c_{k}}{k !}=1 .
$$

This together with the recurrence relation for $c_{k}$ used in the backward direction is an effective technique to generate the $c_{k}$ 's. These data for $\alpha=\beta=0$ are given in column $A$ of Table 2.1. As previously noted in the discussion surrounding (39), simple techniques for getting the $c_{k}$ 's to great accuracy will not as a rule be available in practice. However, asymptotic estimates of the $c_{k}$ 's are relatively easy to get and these are sufficient to estimate errors in interpolation and quadrature processes. For the example at hand, we follow Elliott [12], where $c_{k}$ is written in terms of a contour integral. Then, because $f(z)=(1+z)^{-1}$ has a simple pole at $z=-1$, residue theory is applied to yield

$$
c_{k} \sim \frac{(-)^{k}[2 \pi k(4+3 \sqrt{2})]^{1 / 2}}{(3+2 \sqrt{2})^{k+1}}
$$


and corresponding numerical values are listed in column $B$ of Table 2.1. This result can also be deduced from Luke [11, v. 1, p. 237, Eq. (11)].

TABLE 2.1

\begin{tabular}{rrr}
\multicolumn{3}{c}{$c_{k}$} \\
\cline { 2 - 3 } 0 & \multicolumn{1}{c}{$A$} \\
0 & 0.69314718 & \\
1 & -0.23832462 & -0.21184605 \\
2 & 0.05456674 & 0.05140247 \\
3 & -0.01123996 & -0.01080136 \\
4 & 0.00220464 & 0.00213991 \\
5 & -0.00042037 & -0.00041049 \\
6 & 0.00007869 & 0.00007715 \\
7 & -0.00001454 & -0.00001430 \\
8 & 0.00000266 & 0.00000262 \\
9 & -0.00000048 & -0.00000048 \\
10 & 0.00000009 & 0.00000009
\end{tabular}

We turn now to the interpolation and quadrature formulas, putting $n=2$.

Table 2.2 gives the $x_{k}$ 's and $d_{k, 2}$ 's, while Table 2.3 gives the partial sums in the infinite series representation of $d_{k, 2}-c_{k},(26)$. The necessary values of $V_{j, k}^{(2)}$ are the same as those in Table 1.3.

TABLE 2.2

\begin{tabular}{llr}
$k$ & \multicolumn{1}{c}{$x_{k}$} & \multicolumn{1}{c}{$d_{k, 2}$} \\
0 & $1 / 2-\sqrt{15} / 10$ & 0.69312169 \\
1 & $1 / 2$ & -0.23809524 \\
2 & $1 / 2+\sqrt{15} / 10$ & 0.05291005
\end{tabular}

TABLE 2.3

$$
d_{k, 2}-c_{k} \sim \sum_{i=0}^{s} c_{6+i-k} V_{6+i-k, k}^{(2)}
$$

$\begin{array}{ccccc}s \backslash k & 0 & 1 & 2 \\ 0 & -0.00002597 & 0.00022700 & -0.00165348 \\ 1 & -0.00002597 & 0.00022700 & -0.00165348 \\ 2 & -0.00002549 & 0.00022956 & -0.00165623 \\ 3 & -0.00002549 & 0.00022956 & -0.00165623 \\ 4 & -0.00002549 & 0.00022939 & -0.00165674\end{array}$

To illustrate the interpolation formula and its error, we use (8) and (27). Let $x=1 / 3$. Then $f_{2}(x)=0.75485$ whereas $f(x)=0.75$. Using the first term of (27) and column $A$ of Table 2.1, we get the approximate error -0.00458 . The asymptotic estimate of the remainder obtained using the first term of (27) and column $B$ of Table 2.1 is -0.00440 . This is quite accurate considering the fact that $n$ is small. 
Notice that if one has asymptotic estimates for the $c_{k}$ 's then the coefficients for $k=0,1, \ldots, n$ can be computed more accurately using in (26) the $d_{k, n}$ 's of Table 2.2, asymptotic values for $c_{2 n+2+s-k}$ and the $V_{j, k}^{(n)}$ given in Table 1.3. This gives $c_{0}=0.69314668, c_{1}=-0.23831925$, and $c_{2}=0.05451813$, which is a considerable improvement of the asymptotic estimate to the values given in column $A$ of Table 2.1.

For the quadrature formula (30), the weights $\lambda_{\gamma, 2}^{(0)}$ are $5 / 18,4 / 9$, and $5 / 18$ for $\gamma=0,1,2$, respectively. The approximate integral is 0.69312169 while the exact value is 0.69314718 . Using three terms of (32), we get 0.00002549 , which is the true error. The first term of (32) is 0.00002597 while the asymptotic estimate of the first term, using column $B$ of Table 2.1, is 0.00002546 , either one of which is sufficient to estimate the error.

Mathematics Department

University of Missouri

Kansas City, Missouri 64110

1. Y. L. LUKE, "On the error in a certain interpolation formula and in the Gaussian integration formula," J. Austral. Math. Soc., v. 19, 1975, pp. 196-210.

2. V. I. KRYLOV, Approximate Calculation of Integrals, Fizmatgiz, Moscow, 1959; English transl., Macmillan, New York, 1962. MR 22 \#2002; 26 \#2008.

3. W. BARRETT, "Properties of Gaussian quadrature formulae," Comput. J., v. 3, 1960/61, pp. $272-277$.

4. J. MCNAMEE, "Error-bounds for the evaluation of integrals by the Euler-Maclaurin formula and by Gauss-type formulae," Math. Comp., v. 18, 1964, pp. 368-381. MR 32 \#3264.

5. M. M. CHAWLA \& M. K. JAIN, "Error estimates for Gauss quadrature formulas for analytic functions," Math. Comp., v. 22, 1968, pp. 82-90. MR 36 \#6142.

6. M. M. CHAWLA \& M. K. JAIN, "Asymptotic error estimates for the Gauss quadrature formula," Math. Comp., v. 22, 1968, pp. 91-97. MR 36 \#6143.

7. M. M. CHAWLA, "Asymptotic Gauss quadrature errors as Fourier coefficients of the integrand," J. Austral. Math. Soc., v. 12, 1971, pp. 315-322. MR 45 \#1384.

8. N. K. BASU, "Errors in the representation of a function by Chebyshev polynomials of the second kind," SIAM J. Numer. Anal., v. 10, 1973, pp. 485-488. MR 48 \#538.

9. J. D. DONALDSON \& D. ELLIOTT, "A unified approach to quadrature rules with asymptotic estimates of their remainders," SIAM J. Numer. Anal., v. 9, 1972, pp. 573-602. MR 47 \#6069.

10. P. D. TUAN \& D. ELLIOTT, "Coefficients in series expansions for certain classes of functions," Math. Comp., v. 26, 1972, pp. 213-232. MR $46 \# 598$.

11. Y. L. LUKE, The Special Functions and Their Approximations. Vols. 1, 2, Math. in Sci. and Engineering, vol. 53, Academic Press, New York, 1969. MR 39 \#3039; 40 \#2909.

12. D. L. ELLIOTT, "The evaluation and estimation of the coefficients in the Chebyshev series expansion of a function," Math. Comp., v. 18, 1964, pp. 274-284. MR 29 \#176. 